\title{
Tachyonic Chaos and Causality in the Open Universe
}

\author{
ROMAN TOMASCHITZ \\ Department of Physics, Hiroshima University, Higashi-Hiroshima 724, Japan
}

\begin{abstract}
The chaoticity of classical world lines in extended Robertson-Walker cosmologies is pointed out and related to the fractal limit set of the covering group of the spacelike slices. We investigate the possible existence of tachyons (faster-than-light particles) in this context. The cosmic time and the 3-space co-ordinates comoving with the galactic background provide a distinguished frame of reference. With respect to this comoving frame the causality of tachyonic events can be unambiguously defined, contrary to Minkowski space. We study the dynamics of tachyons and show that tachyonic world-lines are chaotic in the center of the spacelike slices. Copyright (C) 1996 Elsevier Science Ltd.
\end{abstract}

\section{INTRODUCTION}

One of the basic problems one faces in cosmology is that Einstein's equations do not give any hint on the global topological structure of the Universe. This fact, that we do not know the boundary conditions to be imposed on cosmological solutions, was pointed out by the mathematician F. Klein soon after Einstein proposed his first cosmological model, in which the 3-space is closed and has positive constant curvature. Einstein assumed that the topology of the 3-space is that of a 3-sphere, but it can as well have the topology of projective 3-space, without being at odds with the field equations and the homogeneity and isotropy postulates, which are observationally fading today.

It was, however, not until much later that the global behavior of world lines in these topologies was studied, cf. Refs. [1] and [2]. At the end of the fifties general relativists got acquainted with more sophisticated algebraic topology, and in the review [3] it was suggested to try Seifert fiber spaces as spacelike slices in Robertson-Walker (RW) cosmologies. This is essentially what we will do in Section II of this paper.

In the seventies and eighties there was a flurry of activity concerning quantum mechanics, semiclassical mechanics [4], and second quantization [5] on multiply connected manifolds. The spaces considered were mainly toy models of low dimensionality and constant curvature, e.g. Riemann surfaces, or closed Euclidean cosmologies with 3-tori as space sections. The newly emerging field of quantum chaos, in particular Selberg's trace formula on compact Riemann surfaces $[6,7]$, and the chaoticity of geodesic flows $[8,7]$ found a great deal of attention.

Interestingly, in this period general relativity stayed largely unaffected by these developments, though there were some studies on chaos in mixmaster cosmologies [9-12] and on the mixing of geodesic flows in cosmologies with compact hyperbolic 3-slices [13]. The reason for that was perhaps that nonlinear dynamics is based to a large extend on Hamiltonian formalism, whereas general relativity is based on the principle of general and manifest covariance, and it is not always easy to find a common denominator for this. So, for example, the very definition of Lyapunov exponents, which defines the degree of instability in Hamiltonian mechanics, is ambiguous in general relativity, because it is not covariant, it crucially depends on the choice of the time variable. Moreover, I will point 
out in Section II why other well established descriptions of chaoticity in Hamiltonian systems, like mixing or ergodicity, are not really suitable for the problems one faces in cosmology.

Nevertheless, in the nineties it has become more widespread to apply concepts of nonlinear dynamics to general relativity, though I think that the interface of these two disciplines is as yet very little explored. A recent review on the present state of the art in this borderline field is Ref. [14]. Studies of chaos in the gravitational field of charged black holes can be found in Refs. [15] and [16]. In Ref. [17] fractal geometries were investigated in connection with the microscopic structure of spacetime, Banach-Tarski theorem and conjugate complex time.

Let us come back to Seifert fiber spaces as possible candidates for the space sections. As mentioned, Einstein's equations do not determine the topology of the 3-slices, if they are open or closed or their connectivity. We do not even know the local curvature, because we do not know the energy-momentum tensor of the matter content of the Universe. But what we do know is that the galaxies are more or less equidistributed, and that suggests that we should look for a mechanism to create chaos. This in turn suggests that we assume the 3 -slices as locally hyperbolic, because hyperbolicity creates instability [8,6]. The curvature of the 3-space we choose as constant. We are interested in the evolution of the Universe on a global level, and local inhomogeneities of the metric are assumed to be averaged out. The practical reason to do so is that there is just no way to determine a 3-space metric of lesser symmetry, because the energy momentum tensor is unknown. If we agree to take hyperbolic 3-manifolds as spacelike slices it still remains to settle the topology. The question here is not so much what is the topology of the 3-space, but rather how does it evolve. The 3-space should be able to undergo global metrical and also topological deformations, as schematized in Fig. 1, without changing locally the curvature, apart from a rescaling of the curvature radius with the expansion factor (extended RW cosmologies, cf. Refs [18-23]. This requires that the space sections are open - since closed hyperbolic 3-spaces are rigid, they cannot be metrically deformed (Mostow rigidity theorem [24]). Moreover, closed hyperbolic 3-manifolds have rather bizarre topologies [24-26], and it is difficult to motivate why the 3-space should have acquired, once and for all, one in particular of these topologies [13, 27]. Universes with closed multiply connected 3-slices seem also to be at odds with topological censorship ideas [28].

It seems to me much more natural to assume that the Universe is open (i.e. infinite), and dynamically evolving, with transitions from one topology to another. We do not know the laws that determine this cosmic evolution or the current topology, but we can try to find physical effects of a multiply connected topology and of metrical deformations. Evidently we should do this whenever possible without reference to a specific type of topology. So, for example, global metrical deformations of the 3-space create particles in quantum fields and backscattering of electromagnetic waves [29]. They create angular fluctuations in the temperature of the microwave background radiation [22], and the multiple connectivity of the 3-space causes self-interference in wave fields and CP violation [30]. I have recently written reviews on these effects [23, 31], and I shall not report on them further here. As a prerequisite for the tachyonic chaos discussed in this article, I shall give in Section II a sketch of chaos in extended RW cosmologies, and how it relates to the equidistribution of the galactic background.

Sections III-VI are devoted to new endeavors. We shall study faster-than-light particles (tachyons) in the context of the cosmology outlined in Section II. In Refs [32] and [33] tachyons were studied in Minkowski space, and they were found to violate the causality principle. In the theory presented, causality is strictly retained. The cosmic time and the 3-space coordinates comoving with the galactic background provide a distinguished frame of reference. In this frame one can unambiguously define the causality of tachyonic events, 
as well as a positive definite energy for tachyons. Observers, which are moving in coordinate frames in which the cosmic time and the comoving 3-space coordinates are mixed up, may see the time order of events inverted, as an artifact of their frame. We show, cf. Section V, that a geodesically moving tachyon can reach within a finite time the boundary at infinity of the spacelike slices, provided the expansion factor has turning or inflection points. A tachyon can likewise get trapped in the chaotic nucleus of the 3-space, and its trajectory is dense there within a finite time. In Section VI we sketch some open research problems.

\section{THE CHAOTIC NUCLEUS OF AN OPEN UNIVERSE}

As pointed out in the Introduction, there are three conditions to be satisfied that the 3-space admits a dynamical evolution by global metrical deformations, and that space-time itself provides a mechanism to generate chaos, which can account for the uniform galactic background. The 3-space must be open, multiply connected, and locally hyperbolic. Hyperbolicity is necessary to generate the instability of geodesics, and infinite volume and a multiply connected topology are necessary to allow evolution by global deformations, cf. Fig. 1. These deformations of the 3-space do not affect locally the curvature. They do not create local inhomogeneities in the metric. Finally, the multiple connectivity is necessary to confine the unstable world lines to a finite region, so that they can get chaotic there.

I want to outline here, without going into technicalities, why the chaotic nucleus appears, and how one can qualitatively analyze the global behavior of trajectories in the multiply connected 3-space, cf. Refs [34] and [35].

The starting point is the RW line element $\mathrm{d} s^{2}=-c^{2} \mathrm{~d} \tau^{2}+a^{2}(\tau) \mathrm{d} \sigma^{2}$, with $a(\tau)$ the

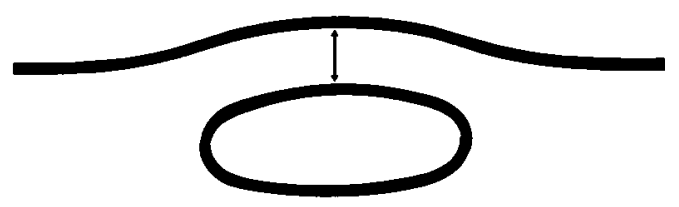

(a)

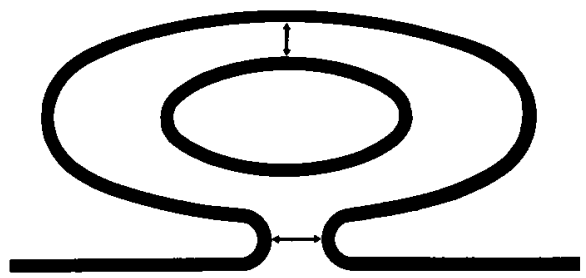

(c)

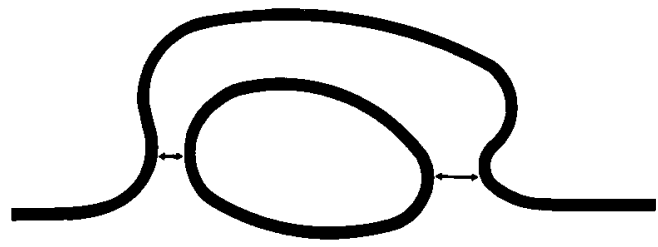

(b)

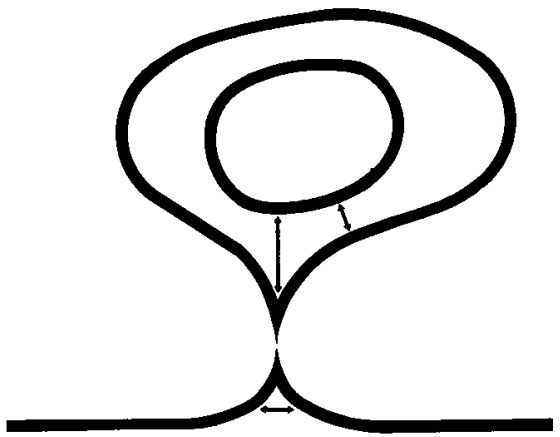

(d)

Fig. 1. (a-d) Global metrical deformations of the 3-space do not change the local curvature. The depicted boundaries are at infinity, unattainable within a finite time for a particle or ray moving with a uniformly bounded speed. In particular the distances indicated by double arrows are infinite. In Fig. 1(d) a bubble (of infinite volume) peels off, leaving behind a cusp singularity, cf. Problem 5 in Sec. VI. 
expansion factor, and $\mathrm{d} \sigma^{2}$ the line element of hyperbolic 3-space $H^{3}$ in some coordinate representation, cf. Section V. The multiply connected 3-space is represented in $H^{3}$ (the covering space) as a non-Euclidean polyhedron $F$, whose faces are lying on hyperbolic planes or on the boundary at infinity of $H^{3}$. The polyhedral faces on hyperbolic planes are identified by elements $\gamma_{i}$ of the invariance group of $H^{3}$, the Lorentz group. The $\gamma_{i}$ and their inverses generate a discrete subgroup $\Gamma$, the covering group of the 3-space, acting on $H^{3}$. If we apply $\Gamma$ to the polyhedron $F$ we get a tiling $\Gamma(F)$ of $H^{3}$ by polyhedral images. This tiling has accumulation points on the boundary at infinity of $H^{3}$, cf. Fig. 2, which constitute the limit set $\Lambda(\Gamma)$ of the covering group. This fractal limit set is of crucial importance in studying geodesic motion on the 3-manifold, since it determines the chaotic center of the 3-space.

Geodesics in the multiply connected 3 -space are obtained by projecting $H^{3}$-geodesics into the polyhedron $F$ by means of the universal covering projection. If an arc $s_{i}$ of the covering geodesic lies in a tile $\gamma_{n}(F)$, then $s_{i}$ is mapped into $F$ by the inverse of $\gamma_{n}$. Thus the trajectory in the 3-space $(F, \Gamma)$ is a collection of $\operatorname{arcs} \gamma_{n}{ }^{-1}\left(s_{i}\right)$. The end points of these arcs, lying on the faces of $F$, are identified by the face-pairing transformations. In this way one gets a smooth trajectory, geodesically looping in the 3-space.

If the covering trajectory has its initial and terminal points in the limit set $\Lambda(\Gamma)$, then its projection consists of infinitely many arc pieces $\gamma_{n}{ }^{-1}\left(s_{i}\right)$, because it intersects infinitely many tiles $\gamma_{n}(F)$ which accumulate at its end points. These arc pieces are confined to a finite domain in the 3-space. This domain can be geometrically defined as the covering projection $C(\Lambda) \backslash \Gamma$ of the hyperbolic convex hull $C(\Lambda)$ of the limit set $\Lambda(\Gamma)$ in $H^{3}$. This confinement and the instability caused by the hyperbolicity of the metric make these trajectories chaotic in the center $C(\Lambda) \backslash \Gamma$ of the 3-space.

If the terminal point of the covering trajectory lies outside $\Lambda(\Gamma)$, then the trajectory will leave the center $C(\Lambda) \backslash \Gamma$, and tend to the boundary at infinity of the 3-space, cf. Fig. 1. If neither the initial nor the terminal point lies exactly in $\Lambda(\Gamma)$, but very close to it, then the covering trajectory will intersect a finite but very large number of tiles, and its projection will shadow chaotic trajectories in $C(\Lambda) \backslash \Gamma$, till it finally leaves the center. This shadowing is a typical feature of hyperbolic dynamical systems [36], with the covering space construction one can make it very explicit and quantitative here.

The center $C(\Lambda) \backslash \Gamma$ is a finite domain, uniquely determined by the topology of the 3-space. It is itself not a differentiable manifold, its surface is infinitely pleated, because $\Lambda(\Gamma)$ is fractal. Such domains are studied by mathematicians for long [37], computer graphical studies of them are not known to me, cf. Problem 1 in Section VI.

Chaoticity is a convincing explanation for the uniformity of the galactic background, but the actual problem is to explain the large-scale inhomogeneities in the galactic distribution. The key to this lies in my opinion in the time evolution of the galactic world lines, and on the shadowing of regular trajectories by chaotic ones. The time parametrization of the world lines depends on the expansion factor, which defines the length unit on the 3-space, like in the traditional text book examples of Robertson-Walker cosmologies, but then also on the metrical deformations that the 3-space undergoes. An explicit analytic example of such deformations can be found in Section 5 of Ref. [23]. Ergodic concepts like mixing and the Bernoulli property, which are commonly used in Hamiltonian dynamics to describe the degree of chaoticity, do not reflect the time-evolution of the system. They are based on the geometric shapes of trajectories of infinite length. Here the question is more what happens in finite times, and with trajectories of finite length. There are also many regular trajectories which enter the chaotic center of the 3-space, and which are shadowed there by chaotic ones. These trajectories will not be dense there, but they may come close to every point, before they ultimately leave the center. The question here is how much time it takes 
Fig. 2(a) and (b). Caption overleaf. 
Fig. 2. (a-c) The horizon at infinity of the Poincare half-space $H^{3}$. A spacelike slice $(F, \Gamma)$ is realized in $H^{3}$ as a polyhedron $F$ with face-identification. The face-pairing transformations generate a discrete group $\Gamma$ which gives, if applied to the polyhedron, a tessellation $\Gamma(F)$ of $H^{3}$ with polyhedral images. This tessallation induces by continuity a tiling on the boundary of $H^{3}$ which is depicted here. The qualitative structure of the fractal limit set $\Lambda(\Gamma)$ depends on the topology of the 3-slices, which is in turn determined by the covering group. For quasi-Fuchsian groups [26] like here, the limit set is a Jordan curve (not self-similar). In all three examples the 3-space fibers over an open interval, with Riemann surfaces $(g=19)$ as fibers. The depicted tilings correspond to 3-slices which are globally non-isometric, but have the same topology and curvature. They make deformations as schematized in Fig. 1(a-c) quantitative. For example, the polyhedral tiling of $H^{3}$ is obtained by placing hemispheres onto the circular arcs. The colors label generations in the tiling procedure [34]. The chaotic trajectories in the 3-space have covering trajectories with initial and terminal points in $\Lambda(\Gamma)$. If the end points are not in $\Lambda(\Gamma)$ but close to it, then the trajectory is regular, but it can shadow a chaotic trajectory over a long time. The convex hull $C(\Lambda)$ of $\Lambda(\Gamma)$ is the intersection of all hyperbolic half-spaces which contain $\Lambda(\Gamma)$. Projected into $(F, \Gamma)$ it constitutes the center $C(\Lambda) \backslash \Gamma$ of the 3-space, see Ref. [29] for an explicit example of this projection. 
to create a reasonably uniform distribution, inhomogeneities will always remain in a finite time, cf. Problems 1 and 3 in Section VI.

\section{SUPERLUMINAL SIGNALS-CAUSALITY-RELATIVITY}

In the sixties, several authors started to scrutinize whether faster-than-light particles can be implemented into the theory of special relativity, cf. Refs [32] and [33], and the review [38]. It is in fact very straightforward to adapt the basic formulas of relativity to this situation, one has just to change some signs in the standard equations. However, in doing so one realizes very soon that there is also a price to pay, for causality is violated.

It is easy to see how this comes about in Minkowski space. Consider two events which are separated in one coordinate frame $(x, \tau)$ by space and time intervals $\Delta x, \Delta \tau$. In a second frame $\left(x^{\prime}, \tau^{\prime}\right)$ with a relative velocity $u<c$ the time separation is $\Delta \tau^{\prime}=$ $\Delta \tau\left(1-v u / \mathrm{c}^{2}\right)\left(1-u^{2} / c^{2}\right)^{-1 / 2}$, with $v:=\Delta x / \Delta \tau$

If the two events correspond to the emission and reception of a superluminal signal, moving with a speed $v>\mathrm{c}^{2} / u$ in frame $(x, \tau)$, then $\Delta \tau^{\prime}$ is negative. This means that in frame $\left(x^{\prime}, \tau^{\prime}\right)$ the reception occurs before the emission, and that the time order of cause and effect is inverted, or if one prefers, that cause and effect are interchanged. This is in flagrant contradiction with our traditional conception of causality, namely that every effect has a cause, that the cause precedes the effect, and that the decision what is cause and what is effect can be unambiguously made, i.e. independently of the observer.

Note that the sign change of the time interval is not a proper relativistic effect. It may occur for arbitrarily small relative speed $u$, if the signal is sufficiently fast, $v>c^{2} / u$. In fact, the Lorentz boost above does not have a uniform Galileian limit, $\Delta \tau^{\prime}=\Delta \tau$, for $u \rightarrow 0$, since $v$ is not bounded from above. Assume an observer who is at - always approximate rest with respect to two events $A, B$, which are connected by superluminal signals whose transmission speed is approaching infinity. Then unavoidable statistical fluctuations in his position with respect to the two events will change the time order in his observations. He will not be able to decide whether the signals move from $A$ to $B$ or vice versa.

To sum up, if a superluminal signal moves in one frame from $A$ to $B$, in other frames it will appear to move from $B$ to $A$, and in some frames it will even not be possible to decide on the initial and terminal point. So, for example, if $A$ presents a car accident, and $B$ obsequies, one observer will see the car accident first, and a signal emitted at $A$ that triggers the obsequies when received at $B$. A second observer will see that time-inverted, like a movie running backwards, namely at first the obsequies at $B$, and a signal emitted from $B$ which seems to trigger the car accident later on at $A$. According to the principle of special relativity, no observers are preferred, all observations in uniformly moving frames have equal physical reality. Therefore, it is not possible to attach in Minkowski space an observer-independent meaning to the statement that event $A$ causes event $B$ by means of the transmission of a superluminal signal, which according to our conception of causality implies that the emission happens prior to the reception at $B$.

It was suggested in Ref. [39] that it would not be logically inconsistent to relax our traditional conception of causality, and to admit that effects can also precede their causes. I think that it is possible that our conception of causality, which is presumably based to a large extend on experience, can break down in extreme limits. If, e.g., signals approach infinite speed, then causality is effectively undecidable even in an individual frame, as pointed out above. But otherwise a simple classical theory of superluminal signals should be in accord with causality, and should not conflict with established laws of physics. Note that in this section we do not make any assumptions about the physics of the signal transfer. Therefore, coming back to our example of car accident and obsequies, a criterion 
is necessary, that the second class of observers that see the car accident caused by the obsequies can convince themselves that their observation is an illusion, an artifact of their frame. The first class of observers, which see the car accident prior to the obsequies, should likewise be able to check that their observations correspond to reality. The introduction of such a criterion obviously violates the principle of relativity, according to which all observers in all uniformly moving frames share equal physical reality.

Apparently, superluminal signals, causality, and the relativity principle do not fit together. At least one of them must be dropped. Einstein excluded the possibility of superluminal signals and kept causality and relativity. The authors in Refs [32, 33 and 39] dropped causality and retained superluminal signals and relativity. In the next section we will drop relativity in a cosmological context, by introducing an absolute, universal frame of reference defined by the galactic background. In this way, we retain causality and the possibility of superluminal particles.

\section{SUPERLUMINAL SIGNALS-CAUSALITY-COSMOLOGY}

In this section we study superluminal signal transfer in the context of an absolute cosmic frame of reference, which is provided by the galactic background and the cosmic time parameter in the expansion factor, which sets the length unit on the 3-slices.

Let us discuss at first how this universal cosmic frame relates to the principles of covariance and general relativity. The principle of general covariance states that all physical equations must be covariant under general co-ordinate transformations, and can, therefore, be written in a form-invariant way, in all co-ordinate frames on the Riemannian 4-manifold. On a multiply connected Riemannian manifold this principle is actually a mathematical necessity. As long as one considers a single co-ordinate chart one can write on it any equation one likes, but if the manifold is multiply connected, it cannot be covered by one single chart, and in this case a differential equation on the manifold must be covariant, otherwise it is ill-defined. So, if we want to use differential equations to describe physical laws in a multiply connected cosmology, they must be covariant to be consistent.

However, physical laws go beyond mathematical equations, and this is what makes the difference between the principles of relativity and covariance. The principle of general relativity states that the same physical laws hold in every co-ordinate frame. With physical laws we mean, for example, Maxwell's equations, but then also that a closed physical system has a well defined energy, that this energy is bounded from below, or that the causality of a physical process is unambiguous.

A space which is homogeneous and isotropic is a mere mathematical abstraction. In reality space can only be perceived by its inhomogeneities. Space in cosmology is the continuum, the vessel, which our imagination builds around the discrete and rather inhomogeneous grid of receding galaxies. But this grid is the essence of space, not the space-time continuum in which we conveniently embed it. It is this unfortunate conception of space as a vessel, which tempts us to assume that the Universe is closed.

General relativity is based on the hypothesis that the metric defining space is in turn determined by the contents of space. But the metric is only a means to make our perception of space and time quantitative, like the coordinate axes of elementary geometry. There is, for example, no reason to define an energy functional for its evolution. Despite being itself a tensor field, the metric is so fundamentally different from real objects like electromagnetic fields, which propagate in the space that it defines.

Now, as long a space is generated in our imagination by rectangular coordinate frames, essentially void, apart from a couple of observers and interacting mass points, a relativity principle seems to be quite natural. However, this type of space is more a category of our 
thinking, a geometric abstraction. In reality, space is not empty, and it is its content which defines it. In the cosmology sketched in Section II it is much less obvious that all frames should have the same physical reality. For example, it is easily possible to define a positive definite energy functional for quantum fields in the galactic frame comoving with the center, cf. Ref. [30]. But there are also frames in which the cosmic time and the comoving spatial coordinates are mixed up, and where we cannot extract from the energy-momentum tensor a quantity that comes even close to energy. Accordingly it is difficult to consent that frames in which we can unambiguously define a positive definite energy functional, and other frames in which we cannot, should be physically equivalent.

Therefore, it seems to me quite natural to introduce an absolute, universal frame of reference, namely the frame in which the observers are comoving, in statistical equilibrium, with the galactic background in the center. General covariance is retained as a mathematical necessity, cf. the preceding discussion. In this frame the laws of physics, in particular causality, are supposed to be valid, it is the frame that exhibits absolute cosmic reality. Observers in other frames obtained by applying arbitrary coordinate transformation to this universal cosmic frame, may well see a distorted cosmic reality, like causality violation or negative energies, just as an artifact of their particular frame of reference which mixes the cosmic time and comoving 3-space coordinates of the universal frame. It is this galactic frame of reference in which we must be able to define unambiguously physical quantities, in which physical laws hold universally true, and in which physical processes evolve causally.

By the introduction of this universal frame every observer has the possibility to evaluate the reality of his observations, just by connecting his frame to the absolute cosmic frame by a coordinate transformation. In particular he can compare the time order of events in his frame to the flow of cosmic time. For example, every observer can introduce a locally geodesic frame in his neighborhood. If a second observer moves uniformly within this neighborhood, then their frames can be approximately linked by a Lorentz boost. Each of the observers can also link his frame to the universal cosmic frame, and has so a criterion to assess the reality of the time order in his frame, cf. the end of Section III.

Remark. In Ref. [33] it was attempted to design a quantum field theory of tachyons in Minkowski space. The vacuum state in this theory is not Lorentz invariant, compare also the discussion in Ref. [40]. In the cosmology presented here the vacuum could be defined with respect to the universal galactic frame of reference without conflicting with general covariance.

\section{TACHYON DYNAMICS IN COSMOLOGY}

Is there any reason to study tachyons [32, 33], hypothetical, superluminally moving particles? If we assume that the Universe is open, then there are indeed reasons to speculate on the existence of faster-than-light particles. In an infinite universe, the existence of a uniform bound on the speed of signal transfer means in a sense that one moves always infinitely slowly, and it is difficult to imagine a Machian network of communication. Moreover, one can question the physical relevance of an open universe on the grounds that we will not be able to verify what is happening at infinity. The existence of tachyons, however, would give us the possibility to literally look at infinity, as we will demonstrate now.

In the following we will define the classical mechanics of tachyons i.e. of superluminal particles in the cosmology introduced in Section II. At first we define tachyons in the universal covering space, and choose there coordinates which give, if projected onto the 
multiply connected 4-manifold, a coordinate representation of the universal frame of reference which is comoving with the galactic center of the multiply connected 3-slices. The formalism is of course covariant, but quantities like energy or velocity we define only with respect to this universal cosmic frame.

As a coordinate representation of the cosmic frame in the covering space we use $\mathbb{R} \times H^{3}$, where $H^{3}$ denotes Poincarés half-space representation of hyperbolic space (with metric $\gamma_{i j}=t^{-2} R^{2} \delta_{i j}$, rectangular half-space coordinates $x_{i}=\left(x_{1}, x_{2}, t\right), t>0 ; R$ is the curvature radius, $H^{3}$ has constant negative curvature $\left.-1 / \mathrm{R}^{2}\right)$. The metric $g_{\mu \nu}$ on $\mathbb{R} \times H^{3}$ is then of the Robertson-Walker type, $g_{00}=-c^{2}, g_{i j}=a^{2}(\tau) \gamma_{i j}, g_{i 0}=0 ; a(\tau)$ is the expansion factor, $\tau$ denotes the cosmic time $[22,30]$.

Next we define the dynamics of tachyons in this covering space. As in the case of geodesically moving subluminal particles, we may start with the Lagrangian $L=g_{\mu v}$ $\mathrm{d} x^{\mu} / \mathrm{d} s \mathrm{~d} x^{\gamma} / \mathrm{d} s$. We have then immediately an integral of motion, $\mathrm{c}^{2}(\mathrm{~d} \tau / \mathrm{d} s)^{2}-\mathrm{g}_{m n}$ $\mathrm{d} x^{m} / \mathrm{d} s \mathrm{~d} x^{n} / \mathrm{d} s=\epsilon$. We may choose the constant $\epsilon$ as \pm 1 or 0 , by rescaling the parameter $s$. Now, $\epsilon=1$ corresponds to subluminal particles, $\epsilon=0$ to light, and it turns out that the choice $\epsilon=-1$ corresponds to tachyons.

For tachyons and subluminal particles alike, we define the energy-momentum vector as $p^{\mu}=m \mathrm{~cd} x^{\mu} / \mathrm{d} s=:\left(\mathrm{c}^{-2} E, \boldsymbol{p}\right)$. Clearly we have $p^{\mu} p_{\mu}=-\epsilon m^{2} \mathrm{c}^{2}$, and we define the velocity $\boldsymbol{v}$ in this frame as usual by parametrizing this hyperboloid, $p=m \boldsymbol{v} \gamma(\epsilon), E=m c^{2} \gamma(\epsilon)$, with $\gamma(\epsilon):=\left(\epsilon\left(1-\boldsymbol{v}^{2} / c^{2}\right)\right)^{-1 / 2}$, and $\boldsymbol{v}^{2}=g_{i j} v^{i} v^{j}$. In particular we have for tachyons $E \sim m \mathrm{c}^{3}|\boldsymbol{v}|^{-1}$, if $|\boldsymbol{v}| \rightarrow \infty$. From the equations of motion we have $(\mathrm{d} \tau / \mathrm{d} s)^{2}=\mathrm{c}^{-2} \epsilon+$ $v^{2} a^{-2}(\tau)$, with a positive integration constant $v^{2}$ related to the energy, $E=$ $m c^{2}\left(\epsilon+c^{2} v^{2} a^{-2}(\tau)\right)^{1 / 2}$. It follows that $a^{2}(\tau) p^{2}=m^{2} c^{4} v^{2}$ and $|v|=c\left(1+\epsilon c^{-2} v^{-2} a^{2}(\tau)\right)^{-1 / 2}$ Clearly, a tachyon can only exist (interact) as long as its energy is positive.

Remark . This definition avoids the introduction of an imaginary or negative mass [32, 33, 38]. If we apply a coordinate transformation to the 4-momentum $p^{\mu}$, it may happen that its first component $\mathrm{c}^{-2} E$ changes its sign if $\epsilon=-1$, i.e. in the tachyonic case. The fact that the energy is not bounded from below in all frames is a matter of great concern when tachyons are studied in the context of the principle of special relativity [32, 33]. It was suggested, borrowing an idea of quantum mechanics, that tachyons with negative energies moving backwards in time should be reinterpreted as having positive energy and moving forward in time [32]. We do not adopt this point of view here. First of all, this reinterpretation does not solve the causality problem. Secondly, this antiparticle concept is totally foreign to classical mechanics, and I think that it is important to develop a theory of tachyons in close analogy to subluminal particles, given that the very assumption of their existence is highly speculative today. As was pointed out above, the introduction of the comoving galactic frame as universal frame of reference renders the fact that in other coordinate frames energy cannot be reasonably defined irrelevant.

It is sufficient to study a trajectory perpendicular to the complex plane, all other trajectories we obtain by applying to it some transformation of the invariance group of $H^{3}$. Its time parametrization is given by $t(\tau)=\exp \left[ \pm \mathrm{c} R^{-1} \int \mathrm{d} \tau a^{-1}(\tau)\left(1+\epsilon \mathrm{c}^{-2} v^{-2} a^{2}(\tau)\right)^{-1 / 2}\right]$. We consider now only tachyons, i.e. $\epsilon=-1$. We assume that $a(\tau)$ is increasing in an interval $\left[\tau_{0}, \tau_{\infty}\right]$, and that $a^{\prime}\left(\tau_{\infty}\right)=0$. The integration constant $v$ which determines the energy we choose as $v=c^{-1} a\left(\tau_{\infty}\right)$. We have $t(\tau) \sim$ const. $\left|\tau_{\infty}-\tau\right|^{ \pm \alpha},|v| \sim \alpha R a\left(\tau_{\infty}\right)\left|\tau_{\infty}-\tau\right|^{-1}$, for $\tau \rightarrow$ $\tau_{\infty}$, with $\alpha:=c R^{-1}\left|a^{\prime \prime}\left(\tau_{\infty}\right) a\left(\tau_{\infty}\right)\right|^{-1 / 2}$. If in addition $a^{\prime \prime}\left(\tau_{\infty}\right)=0$, we obtain $t(\tau) \sim$ const. $\exp \left( \pm 2 \beta\left|\tau_{\infty}-\tau\right|^{-1 / 2}\right),|\boldsymbol{v}| \sim \beta R a\left(\tau_{\infty}\right)\left|\tau_{\infty}-\tau\right|^{-3 / 2}$, with $\beta:=3^{1 / 2} c R^{-1}\left|a^{(3)}\left(\tau_{\infty}\right) a\left(\tau_{\infty}\right)\right|^{-1 / 2}$.

Let us discuss that a little. If $a\left(\tau_{\infty}\right)$ is a turning or inflection point, and if we choose the initial energy as $E_{0}=m c^{2}\left(a^{2}\left(\tau_{\infty}\right) a^{-2}\left(\tau_{0}\right)-1\right)^{1 / 2}$, then we have $t\left(\tau_{\infty}\right)=0$ or $\infty$. This means that the tachyon, starting at some point in the interior of $H^{3}$ reaches within a time 
$\Delta \tau=\tau_{\infty}-\tau_{0}$ the boundary at infinity of $H^{3}$. In other words, the tachyon can reach within a finite time $\Delta \tau$ every given point in the covering space, however remote from the starting point, and it arrives with a finite fraction of its initial energy there. At any rate it can transfer signals. The same holds true for the 3-space. In this context, a remark on the energy transfer particle-tachyon is appropriate. We do not know the interaction, and therefore the simplest thing is to assume elastic head-on collisions, which are entirely determined by energy-momentum conservation. Interaction is of course only then possible if the energy of the outgoing tachyon is positive, and the energy of the outgoing particle is larger than its rest energy. So, for example, if the incoming momenta of tachyon and subluminal particle are directed against each other, and $p>m_{\mathrm{t}} \mathrm{c}, p$ the incoming particle momentum, $m_{\mathrm{t}}$ the tachyon mass, then interaction is always possible. If the energy of the incoming tachyon approaches infinity (and therefore its velocity the speed of light), then almost all the tachyon energy is transferred to the outgoing subluminal particle. On the other hand, if $E_{t}$ approaches zero, then a fraction of the energy of the particle is transferred to the tachyon, so that both particle and tachyon slow down. In fact, a tachyon looses energy if it speeds up, which is reasonable, for an infinite velocity means in a sense that it can be everywhere at the same time, and then it should not carry energy.

Assume now that a covering trajectory has its initial point somewhere in $H^{3}$ and its terminal point in the limit set $\Lambda(\Gamma)$ of the covering group $\Gamma$ of the multiply connected 3 -space, cf. Section II. The tachyon reaches this limit point within a finite time $\Delta \tau$. We project this trajectory into the 3-space, as sketched in Section II. The projected arcs inherit the time parametrization of the covering trajectory. The projected trajectory enters the center of the 3-space, and becomes dense there within $\Delta \tau$. The hyperbolicity of the metric and the confinement to a finite region make the geodesics chaotic there.

The difference between geodesically moving sub- and superluminal trajectories is in their time parametrization, not in their shape, at least as long as we neglect global deformations of the 3-space within $\Delta \tau$. The world-line of a subluminal particle in the center has of course only a finite length within $\Delta \tau$, it can come close to every point, but it is not really dense there.

\section{CONCLUDING REMARKS, AND SOME OPEN RESEARCH PROBLEMS}

I have explained in the Introduction my approach to cosmology, and the reasons to study a cosmology whose spacelike sections are open, and whose evolution is very different from the traditional cosmological solutions of Einstein's equations. I have likewise pointed out in Section $\mathrm{V}$ the motivation to consider the existence of superluminal signal transfer in the context of an infinite universe. I conclude with a critical comment on the utility of Einstein's equations in cosmology.

If one compares the two reviews [3,41] on theoretical cosmology, separated by thirty-five years, one can only be surprised by the change of emphasis and style, because in this period there have not been any major changes in the foundations of Einsteinian cosmology. Whilst the authors of Ref. [3] tried to establish a coherent cosmic world view, the trend today goes to classify cosmological solutions of Einstein's equations like species in zoology.

If one speaks about cosmological solutions one must keep in mind that these are not solutions of a given system of equations. One starts with a more or less symmetric metric, calculates the Einstein tensor, and defines so a more or less isotropic and homogeneous energy-momentum tensor. In this way there are many cosmological solutions known, and classified according to their symmetries. None of them is particularly distinguished, and their predictive power is according to their construction poor. The trouble with this approach to cosmology is, like with the many-worlds interpretation of quantum mechanics, 
that there is only one Universe, only one World. Even if the 3-space is disconnected, one would have to regard its components as a unity. A good cosmology has to provide a basis for that, and to explain how it came about, and what are the consequences for the individual components.

In the following, I indicate several open research problems in extended RW cosmology, their context is discussed in Section II. With each topic I will roughly indicate the technical steps to be carried out.

\section{CHAOS IN THE CENTER OF AN OPEN AND MULTIPLY CONNECTED RW COSMOLOGY: FORMATION OF INHOMOGENEITIES IN THE GALACTIC BACKGROUND}

I discuss at first the static case, i.e. the topology and the metric of the 3-space is time independent, apart from a rescaling of the hyperbolic 3-space with the expansion factor. No global metrical deformations take place. The problem is here to explicitly study the time evolution of world lines in the center of the 3-space, and to investigate the mixing in a finite time. One has to study how long it takes to become a trajectory effectively dense, and if islands appear which are never or rarely crossed by the trajectory. Likewise, one must ask how efficiently are regular trajectories shadowed by chaotic ones over a finite time. If one starts with an arbitrary asymmetric distribution of mass points, and if one chooses initial conditions which result in chaotic or nearly chaotic trajectories, then these mass points will tend to become at first nearly equidistributed in the center, but ultimately they will start to diffuse out into the open 3-space. This diffusion takes place in practice, because in order to confine a trajectory to the center for all times, one has to choose the initial conditions with infinite precision. One has to study the time evolution, and to figure out which inhomogeneities (clusters, voids) appear, when they appear, and on which scales.

This is essentially a numerical, computer graphical problem. It is quantitatively solved by means of the covering space construction. The covering group and its limit set have to be calculated. Then one calculates the convex hull of this set in the covering space, and finally one projects this convex hull into the 3-manifold with the covering projection. One obtains so the center of the 3-space. The most complicated step here is to obtain the convex hull of the fractal set. One uses as covering space the Klein model of hyperbolic geometry [42], because Euclidean convexity coincides with hyperbolic convexity there. In studying the chaoticity of trajectories one slices the 3-space into 2-sections similar to Poincaré sections in Hamiltonian dynamics, but this is real space here. Some computer simulations which give an impression of the efficiency of the mixing mechanism on compact Riemann surfaces can be found in Ref. [7].

\section{THE INFLUENCE OF GLOBAL METRICAL DEFORMATIONS ON THE GALACTIC DISTRIBUTION AND THE BACKGROUND RADIATION}

We have two time scales to take into account. Cosmic time is defined by the expansion, which is the relevant time of an observer comoving with the galactic background. The second time scale is that on which metrical deformations take place. In fact, only if this second scale is much larger, if the deformations are sufficiently adiabatic, can one properly speak of a uniform expansion. If these scales approach each other, the expansion of the 3 -space will become inhomogeneous, though the 3-space stays constantly curved. As to world lines, a deformation of the 3-space will influence both their geometric shape and their time parametrization, but modestly, if the deformation is adiabatic. In the case that 
expansion and deformation happen on the same scale, this will not change the chaoticity of trajectories in the now time dependent center, but it will change their shape and their time parametrization. The mixing, which occurs merely because the trajectories are unstable and confined, will always tend to create an equidistribution, but now no longer a statistical equilibrium.

If one technically realizes a global deformation, one has to take care that the 3-space stays of constant curvature and the 4-manifold stays a 4-manifold. In order that the second requirement is satisfied, the covering group of the 3-space must be time-independent. Only the metric in the covering space may vary in time, otherwise one cannot attach a time-axis to the 3-manifold. The 3-space should stay constantly curved. So the time variation is generated by a time-dependent coordinate transformation of the hyperbolic metric in the covering space. But this diffeomorphism of the covering space must be chosen so that it is compatible with the covering group. If the diffeomorphism is applied to the covering group, the transformed group must stay the covering group of a hyperbolic manifold, or better of a sequence of time-parametrized hyperbolic 3-manifolds, cf. Problem 5 below. This is a strong restriction on possible deformations, they can be parametrized by a finite number of parameters if the genus of the fibers of the 3-manifold is finite, cf. Ref. [43].

Further restrictions on the size of this parameter space can be obtained by Einstein's equations. In the static case, cf. Problem 1, Einstein's equations on the multiply connected manifold are the same as in the simply connected covering space, because the covering group is a subgroup of the invariance group of the hyperbolic metric, and the energymomentum tensor depends only on the expansion factor and the hyperbolic metric of the 3 -space. The question is now if global deformations can render the pressure or the energy density negative. If so, this would give restrictions on the size and speed of possible deformations. So, for example, this could prevent the 3-space from breaking apart, from getting disconnected, cf. Problem 5. However, Einstein's equations would not give a reduction of the dimension of the parameter space, because sufficiently adiabatic deformations result only in an analytic perturbation of the energy-momentum tensor, which will not change the positivity of pressure and energy. (The energy-momentum tensor is of course defined here by the metric and the Einstein tensor.)

By means of the eikonal equation one can obtain semiclassical frequency shifts of photons, which are induced by adiabatic deformations. They cause angular variations in the temperature of the background radiation [44, 22, 31, 45], and it would be of great interest to have quantitative estimates relating the time evolution of deformations to the size of these now observable angular fluctuations [46].

\section{CLASSICAL DISPERSION IN EXTENDED RW COSMOLOGY}

The task is to develop a probabilistic continuum description of the geodesic chaoticity. In Ref. [47] the concept of a horospherical geodesic flow of expanding bundles of parallel world lines was introduced in a cosmology with a simply connected open 3-space. The world lines are unstable there, but not chaotic. An invariant measure and a covariant evolution equation for the probability density on which this expanding flow acts was constructed, and the dispersion of the density was studied. In Hamiltonian dynamics this instability of the world lines is expressed by Lyapunov exponents, which give the degree of local exponential separation of neighboring trajectories. Lyapunov exponents depend heavily on the time parametrization of the trajectory, which is determined by the expansion factor if we take cosmic time as the relevant time variable. It would be highly desirable to have a geometric and covariant characterization of the geodesic instability, which does not involve time. The point here is that, depending on the expansion factor, the separation 
need not be exponential at all in the cosmic time. As a consequence we found that the dispersion of the density on which the geodesic flow acts can be rather slow, far from being exponential in time. What I suggest now is to study dispersion in the center of the 3-space, where the flow acting on the density is chaotic. On the other hand chaoticity does not depend on the time parametrization of trajectories, but on their geometric shapes in 3-space, see the end of Section II. Due to the mixing mechanism dispersion will be much more rapid there. The dispersion will not be confined to the center, for reasons which I indicated in Problem 1, the density will start to diffuse out of it. After a short time it is in the asymptotic regime, which will be largely independent of the initial distribution, because mixing took place. It would be interesting to study which kinds of inhomogeneities appear in the distribution.

\section{CHAOS IN GRAVITATIONAL WAVES IN THE CENTER OF THE 3-SPACE}

The typical problem of quantum chaos is to relate, basically by semiclassical approximation techniques, wave fields to chaotic trajectories. Analogously, I suggest to study classical linearized gravitational waves, and how they relate to chaotic classical rays via the eikonal approximation. In other words, how the geodesic chaoticity manifests itself in the evolution of linearized gravitational waves. Such problems are fairly difficult to study in Hamiltonian mechanics [6], here it is much simpler. The dynamics in the covering space is integrable, the chaoticity is generated by the projection into the 3-space. This projection is easy to carry out, algorithmically, by periodizing solutions of the wave equation with the covering group, cf. Ref. [23]. A very interesting problem in this connection is also to study the second quantization of free gravitational waves in the multiply connected open 3-space. It would be interesting to investigate whether the Planck distribution is the only possible equilibrium distribution of gravitational radiation, because there is not only one obvious way to carry out the thermodynamic limit (box-quantization), if the space is multiply connected, cf. Ref. [22].

\section{TOPOLOGY CHANGES}

The task here is to model topology changes quantitatively. This can be done in two steps. First we study topology changes of the 3-space as a manifold in its own right. The second step is then to represent these changes in a way that one can extend the 3-manifold to a 4-manifold, by attaching a time axis. As to the first step, topology changes are a result of global metrical deformations. The polyhedron representing the 3-manifold in the covering space undergoes time variations, and so does the covering group. Let us try to model a situation in which the genus of the fibers of the 3-space changes. Such changes can be implemented by topological surgery on the fibers, the difficulty here is to do that continuously. So, for example, if the fibers are Riemann surfaces with a parabolic puncture, then one breaks up the parabolic cusp, and inserts a hyperbolic cycle. On the polyhedron in the covering space this means that one separates the two hyperbolic planes which are tangent and represent the cusp singularity, and inserts four polyhedral faces, which present a topological handle. The whole has to be done continuously, the radius of the base circles of the inserted hyperbolic planes increases continuously in time, starting at zero. Some glimpses on this can be found in Ref. [35]. The hyperbolic metric on the covering space remains unchanged and is induced onto the polyhedron. In order that this sequence of hyperbolic 3-manifolds, parametrized by time, can be used as spacelike slices, one has to transfer the time dependence of the covering group and the polyhedron to the 
metric by means of a diffeomorphism of the covering space, as explained in Problem 2. This is the second step.

This is also a good example of how topological singularities can emerge on the 3-space by global deformations. Note that the metric stays constantly curved on the whole 3-space, the singularity is at infinity. In this case a topological handle is contracted into a topological cusp singularity. Such singularities also appear when the 3-space disintegrates, then the handle does not disappear, but peels off, and gets itself an open hyperbolic 3-manifold with a cusp singularity. It would be interesting to study what happens to quantum fields in this situation. Topology changes have also been considered from a quantum theoretical point of view in Ref. [48].

If one does cosmology in the context of a Riemannian space, one has to deal with its global structure, i.e. with the dynamics of its topology. As mentioned in Section I, we do not know the laws of this dynamics. What we point out here, and what we want to make explicit is that in cosmology the topology itself is a dynamic object, like the metric in local problems of general relativity.

Acknowledgements - The author acknowledges the support of the Japan Society for the Promotion of Science, Contract No. P-95378, and in particular the stimulating atmosphere and kind hospitality of the Particle Physics Group here at Hiroshima University.

\section{REFERENCES}

1. L. Infeld and A. Schild, Phys. Rev. 68, 250 (1945).

2. E. Schrödinger, Expanding Universes. Cambridge University Press (1956).

3. O. Heckmann and E. Schücking, Handbuch d. Physik 53, 489 (1959).

4. N. Hurt, Geometric Quantization in Action. Reidel, Dordrecht (1983).

5. N. Birrell and P. C. Davies, Quantum Fields in Curved Space. Cambridge University Press (1982).

6. M. Gutzwiller, Chaos in Classical and Quantum Mechanics. Springer, New York (1990).

7. N. Balazs and A. Voros, Phys. Rep. 143, 109 (1986).

8. V. I. Arnold, Mathematical Methods of Classical Mechanics. Springer, New York (1978).

9. C. Misner, in Magic, without Magic, edited by R. Klauder. Freeman, San Francisco (1972).

10. C. Misner, in Deterministic Chaos in General Relativity, edited by D. Hobill. Plenum, New York (1994).

11. D. Chitre, Phys. Rev. D6, 3390 (1972).

12. J. D. Barrow, Phys. Rep. 85(1) (1982).

13. C. Lockhart, B. Misra and I. Prigogine, Phys. Rev. D25, 921 (1982).

14. D. Hobill, A. Burd and A. Coley (eds), Deterministic Chaos in General Relativity. Plenum, New York (1994).

15. G. Contopoulos, Proc. Roy. Soc. A435, 551 (1991); ibid. A431, 183 (1990).

16. Y. Sota, S. Suzuki, and K. Maeda, in Dynamical Systems and Chaos. Tokyo (1994), Y. Aizawa et al., (eds). World Scientific, Singapore (1995).

17. M. S. El Naschie, Chaos, Solitons \& Fractals 2, 211 (1992), ibid. 4, 2121 (1994), ibid. 5, 1503 (1995), ibid. 5, 1551 (1995).

18. R. Tomaschitz, J. Math. Phys. 32, 2571 (1991).

19. R. Tomaschitz, Complex Systems 6, 137 (1992).

20. R. Tomaschitz, in Chaotic Dynamics: Theory and Practice, edited T. Bountis. Plenum, New York (1992).

21. R. Tomaschitz, in Quantum Chaos-Quantum Measurement, edited by P. Cvitanovic et al. Kluwer, Dordrecht (1992).

22. R. Tomaschitz, J. Math. Phys. 34, 3133 (1993).

23. R. Tomaschitz, in Deterministic Chaos in General Relativity, edited by D. Hobill. Plenum, New York (1994).

24. W. Thurston, Bull. Am. Math. Soc. (New Series) 6, 357 (1982).

25. S. Krushkal, B. Apanasov and N. Grusevskii, Kleinian Groups and Uniformization in Examples and Problems. Am. Math. Soc., Providence, R.I. (1986).

26. B. Maskit, Kleinian Groups. Springer, New York (1986).

27. G. F. Ellis and R. Tavakol, in Deterministic Chaos in General Relativity, edited by D. Hobill. Plenum, New York (1994).

28. J. Friedman, K. Schleich and D. Witt, Phys. Rev. Lett. 71, 1486 (1993).

29. R. Tomaschitz, Intern. J. Theoret. Phys. 33, 353 (1994).

30. R. Tomaschitz, J. Math. Phys. 35, 1573 (1994).

31. R. Tomaschitz, in Dynamical Systems and Chaos. Tokyo (1994), edited by Y. Aizawa et al. World Scientific, Singapore (1995). 
32. O. Bilaniuk, V. Deshpande and E. Sundarshan, Am. J. Phys. 30, 718 (1962).

33. G. Feinberg, Phys. Rev. 159, 1089 (1967).

34. R. Tomaschitz, Physica D34, 42 (1989).

35. R. Tomaschitz, Intern. J. Theoret. Phys. 31, 187 (1992).

36. R. Bowen, J. Diff. Eq. 18, 333 (1975).

37. D. B. Epstein and A. Marden, in Analytical and Geometrical Aspects of Hyperbolic Space, edited by D. B. Epstein. Cambridge University Press (1987).

38. E. Recami and R. Mignani, Riv. Nuovo Cimento 4, 209 (1974).

39. R. Newton, Phys. Rev. 162, 1274 (1967).

40. M. Arons and E. Sundarshan, Phys. Rev. 173, 1622 (1968).

41. M. MacCallum, in Deterministic Chaos in General Relativity, edited by D. Hobill. Plenum, New York (1994).

42. A. F. Beardon, The Geometry of Discrete Groups. Springer, New York (1982).

43. L. Bers, Ann. Math. 91, 570 (1970).

44. R. Sachs, and A. Wolfe, Astrophys. J. 147, 73 (1967).

45. R. Tomaschitz, in Proceedings of the 7th Marcel Grossman Meeting, Stanford 1994, edited by R. Ruffini and E. Keiser. World Scientific, Singapore (1995).

46. G. Smoot et al., Astrophys. J. 396. L1 (1992).

47. R. Tomaschitz, J. Math. Phys. 34, 1022 (1993).

48. J. A. Wheeler, in The Physicist's Conception of Nature, edited by J. Mehra. D. Reidel, Dordrecht (1973). 